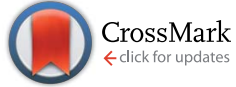

Cite this: J. Mater. Chem. A, 2017, 5, 4103

Received 19th November 2016

Accepted 18th January 2017

DOI: $10.1039 / c 6 t a 09999 h$

www.rsc.org/MaterialsA

\section{Surfactant-exfoliated 2D hexagonal boron nitride (2D-hBN): role of surfactant upon the electrochemical reduction of oxygen and capacitance applications $\dagger$}

\author{
Aamar F. Khan, ${ }^{a}$ Michael P. Down, ${ }^{a}$ Graham C. Smith, ${ }^{b}$ Christopher W. Foster ${ }^{a}$ \\ and Craig E. Banks*a
}

Surfactant-exfoliated 2D hexagonal boron nitride (2D-hBN) nanosheets are fabricated using the surfactant sodium cholate in aqueous media and are explored towards the electrochemical reduction of oxygen (oxygen reduction reaction) within acidic media for the first time. Large quantifiable voltammetric signatures are observed at significantly reduced potentials compared to traditional graphitic-based electrodes indicating 2D-hBN's possible electrocatalytic activity towards the oxygen reduction reaction, therefore having the potential as a useful electrode platform within fuel cell technology. We also demonstrate, for the first time, that surfactant-exfoliated $2 \mathrm{D}-\mathrm{hBN}$ is an effective electrochemical supercapacitor material with a specific capacitance value of up to $1745 \mathrm{~F} \mathrm{~g}^{-1}$. A full analysis of the electrochemical properties of $2 \mathrm{D}-\mathrm{hBN}$ is performed, including the application of a novel capacitive circuit applied to galvanostatic charge/discharge analysis, which provides an unambiguous analysis of the capacitance of the 2D-hBN. Furthermore, a diverse range of methods are introduced and utilised to calculate the specific capacitance, a substantially overlooked and misinterpreted parameter within the literature allowing standardisation in the academic literature to be achieved. In both examples, we demonstrate through control experiments in the form of surfactant modified graphite electrodes, sodium cholate is the major contributing factor to the aforementioned electrocatalytic and capacitive behaviour, which has yet to be reported.

\section{Introduction}

The continuing depletion of natural energy resources has resulted in the need to find alternative means of energy production. ${ }^{1-3}$ Reassuringly, reliance upon finite sources of energy is reducing and alternative means of energy generation are highly sought. One such example is the proton exchange membrane (PEM) fuel cell, in which the oxygen reduction reaction (ORR) occurs, ${ }^{4}$ and this technology currently leads the transportation fuel cell market. However, an increase in the efficiency of PEM fuel cells is highly desirable, therefore research efforts are directed towards improving their performance in terms of power output, efficiency, and longevity. ${ }^{5}$

A significant drawback of PEM fuel cells is the ORR, which can limit fuel cell lifetimes in many devices due to electrode

${ }^{a}$ Faculty of Science and Engineering, Manchester Metropolitan University, Chester Street, Manchester M1 5GD, UK. E-mail: c.banks@mmu.ac.uk; Web: http://www. craigbanksresearch.com; Fax: +44 (0)1612476831; Tel: +44 (0)1612471196

${ }^{b}$ Faculty of Science and Engineering, Department of Natural Sciences, University of Chester, Thornton Science Park, Pool Lane, Ince, Chester CH2 4NU, UK

$\dagger$ Electronic supplementary information (ESI) available. See DOI: $10.1039 /$ c6ta09999h fouling. ${ }^{6}$ The ORR occurs at the cathode of PEM fuel cells, where fuel cell degradation ${ }^{7}$ is synonymous with the ORR due to the formation of hydrogen peroxide $\left(\mathrm{H}_{2} \mathrm{O}_{2}\right)$ when using catalysts that reduce oxygen via the two electron pathway. ${ }^{8}$ Thus, a platinum $(\mathrm{Pt})^{9}$ electrocatalyst is commonly employed as its mechanism for the reduction is a direct four electron pathway that does not produce harmful by-products such as $\mathrm{H}_{2} \mathrm{O}_{2}{ }^{5,10,11}$ However, due to Pt being sparse and expensive, ${ }^{6}$ it is unsuitable for mass production as a fuel cell catalyst. Consequently, research is directed towards cheaper nonprecious metal catalysts $^{12}$ and more pertinently, metal-free carbon based materials. ${ }^{10}$

Previous studies have utilised 2D materials towards the ORR. Qazzazie et al. ${ }^{13}$ observed that nitrogen doped graphene (GN) modified glassy carbon (GC) electrodes resulted in a decrease in activation potential for the ORR in alkali conditions. The potential for the ORR to occur shifts from $-250 \mathrm{mV}$ to $-160 \mathrm{mV}$ upon the GN modification. ${ }^{13}$ Other 2D materials such as $\mathrm{MoS}_{2}$ have been utilised for the ORR. Rowley-Neale et al. ${ }^{14}$ showed that the immobilisation of $\mathrm{MoS}_{2}$ upon a screen-printed electrode (SPE) reduced the potential required for the ORR to occur from $-1000 \mathrm{mV}$ (bare electrode) to $-180 \mathrm{mV}$ (modified 2D-MoS 
electrode) in acidic conditions. ${ }^{14}$ Zhao et $a l .{ }^{15}$ have demonstrated that $\mathrm{MoS}_{2}$ modified GC electrodes result in the ORR occurring at $-420 \mathrm{mV}$ within alkaline conditions. The same report also demonstrated GN GC electrodes resulted in the ORR occurring at $-310 \mathrm{mV} .{ }^{15}$ The lowest potential for the ORR to occur was observed when $\mathrm{MoS}_{2}$ and GN combined resulting in an activation potential for the ORR of $-230 \mathrm{mV} .^{15}$

A lesser researched material is $2 \mathrm{D}$ hexagonal boron nitride (2D-hBN). Zhao et al. ${ }^{\mathbf{1 6}}$ studied the ORR and computationally demonstrated via density functional theory (DFT) that carbonsingly doped into h-BN nanosheets can cause high spin density and charge density resulting in the enhancement of $\mathrm{O}_{2}$ adsorption. ${ }^{16}$ Uosaki et al. ${ }^{6}$ investigated the ORR and immobilised 2D-hBN upon gold substrates/electrodes. Their work elegantly demonstrated that an excellent $2 \mathrm{D}-\mathrm{hBN} /$ gold interaction is observed due to the binding of the $2 \mathrm{D}-\mathrm{hBN}$ monolayer with transition metal surfaces given that the $\mathrm{N}-\mathrm{p}_{z}$ and $\mathrm{B}-\mathrm{p}_{z}$ orbitals of $2 \mathrm{D}-\mathrm{hBN}$ mix with $\mathrm{d}_{z^{2}}$ metal orbitals. ${ }^{6}$ Thus upon modification of a gold electrode with $2 \mathrm{D}-\mathrm{hBN}$, the potential required for the ORR was offset by $c a .0 .27 \mathrm{~V}$ compared to a bare gold electrode. ${ }^{6}$ This report demonstrates that $2 \mathrm{D}$-hBN potentially possesses an electrocatalytic behaviour towards the ORR on this substrate. Additionally, gold is a precious metal with a high cost, making it an expensive substrate and therefore not highly desirable in the design, commercialisation and massproduction of fuel cells. ${ }^{6}$ We have previously reported that surfactant-free (pristine) 2D-hBN nanosheets exhibit an electrocatalytic behaviour towards the ORR when immobilised upon SPEs. ${ }^{17}$ Pristine $2 \mathrm{D}$-hBN reduced the potential required for the ORR to occur by $c a$. $0.28 \mathrm{~V}$ in comparison to unmodified/ underlying SPEs. ${ }^{17}$ Interestingly, no such beneficial response was observed for pristine 2D-hBN when it was modified/ supported on GC and boron doped diamond (BDD) underlying electrodes. ${ }^{17}$ The observed beneficial response at pristine 2D-hBN modified SPEs was attributed to the interaction between 2D-hBN and the roughness of the underlying SPE, whereas the comparatively smooth surface of a GC electrode hindered this. ${ }^{\mathbf{1 7}}$

Another key are within the energy sector is improving the overall power of energy storage devices. A fundamental high power solution is to utilise advanced electrode materials within supercapacitor devices. Supercapacitors are passive and static electrical energy storage devices, utilised in applications from portable electronics to hybrid cars. They are typically seen as a viable future solution in the search for greener and higher powered electronics. Furthermore, supercapacitors are required to store energy whilst offering transient, but extremely high powers, delivered over a short period of time. ${ }^{18,19}$ Thus, developing an improved capacitor performance and a greater understanding/insight into their fabrication and mechanism is of extremely high significance. ${ }^{\mathbf{1 , 2 0}}$

Current research is directed towards chemically doping novel carbon-based nanomaterials with foreign atoms in order to obtain superior properties and impart improved supercapacitor performances. Pertinent materials of note which offer beneficial performances, such as a high a specific capacitance $\left(C_{\mathrm{s}}\right)$, include: vertically aligned boron carbon nitride $(\mathrm{BCN})$ nanotubes (ca. $\left.321 \mathrm{~F} \mathrm{~g} \mathrm{~g}^{-1}\right) ;^{21}$ three-dimensional nitrogen and boron co-doped graphene aerogels ( $\left.c a .239 \mathrm{~F} \mathrm{~g}^{-1}\right)$; $^{22}$ nitrogendoped 2D graphene (ca. 220, and ca. $\left.197 \mathrm{~F} \mathrm{~g}^{-1}\right) ;^{23,24}$ and boron-doped 2D graphene ( $\left.c a .173 \mathrm{~F} \mathrm{~g}^{-1}\right) .{ }^{25}$ Furthermore Gao et $a l^{26}$ have utilised h-BN nanoparticles (in bulk) to form a composite supercapacitor material with reduced-graphene oxide (r-GO). ${ }^{26}$ Their report showed that the h-BN/r-GO composite material offers a $C_{\mathrm{s}}$ value of $140 \mathrm{~F} \mathrm{~g}^{-1}$ when mixed with acetylene black/polyvinylidene fluoride and pressed onto a nickel foam electrode in a $6 \mathrm{M} \mathrm{KOH}$ solution. However, 2D$\mathrm{hBN}$ per se has yet to be explored as a supercapacitor material.

We consider for the first time 2D-hBN nanosheets fabricated using the surfactant sodium cholate in aqueous media and explore the electrochemical reduction of oxygen (ORR) and capacitive performance within acidic media. Furthermore, control experiments, in the form of surfactant modified graphitic electrodes are implemented in order to deconvolute the true electrochemical response. The effect of surfactantexfoliated 2D-hBN, to the best of our knowledge, has yet to be explored towards the ORR and capacitance applications.

\section{Experimental section}

All chemicals utilised were of analytical grade and were used as received from Sigma-Aldrich without any further purification. All solutions were prepared with deionised water of resistivity not less than $18.2 \mathrm{M} \Omega \mathrm{cm}$ and (when necessary) were vigorously degassed prior to electrochemical measurements with high purity, oxygen free nitrogen.

Voltammetric measurements were performed using an 'Autolab PGSTAT 101' (Metrohm Autolab, The Netherlands) potentiostat. Measurements for the ORR were conducted, using a conventional three electrode system. Capacitance measurements, utilising a two electrode system, were undertaken via a method outlined by Kampouris et al. ${ }^{19}$ full details of which can be found in the ESI. $\dagger$

The working electrodes utilised throughout were a screen printed graphite electrode (SPE), which are shown in ESI Fig. 5. $\dagger$ 2D-hBN modified electrodes were prepared by drop-casting aliquots of the desired 2D-hBN solution onto the required working electrode with a micropipette. After 30 minutes the solvent completely evaporated (at ambient temperature) and the modified electrodes were then ready for use. Where necessary, a platinum wire and a saturated calomel electrode (SCE) were used as counter and reference electrodes respectively.

The screen-printed graphite electrodes (SPEs) utilised throughout this work consist of a graphite working electrode, a graphite counter electrode and an $\mathrm{Ag} / \mathrm{AgCl}$ reference electrode. The SPEs, which have a $3 \mathrm{~mm}$ diameter working electrode, were fabricated in-house with appropriate stencil designs using a microDEK 1760RS screen-printing machine (DEK, Weymouth, UK). This SPE design has been previously reported. ${ }^{27-29}$ For the case of each fabricated electrode, first a graphite ink formulation (Product Code: C2000802P2; Gwent Electronic Materials Ltd, UK), which is utilised for the efficient connection of all three electrodes and as the ink material for both the working and counter electrodes, was screen-printed 
onto a polyester (Autostat, $250 \mu \mathrm{m}$ thickness) flexible film. After curing the screen-printed graphite layer in a fan oven at $60{ }^{\circ} \mathrm{C}$ for 30 minutes, an $\mathrm{Ag} / \mathrm{AgCl}$ pseudo reference electrode was included by screen-printing $\mathrm{Ag} / \mathrm{AgCl}$ paste (Product Code: C2030812P3; Gwent Electronic Materials Ltd, UK) onto the polyester substrates, which was subsequently cured once more in a fan oven at $60{ }^{\circ} \mathrm{C}$ for 30 minutes. Finally, a dielectric paste (Product Code: D2070423D5; Gwent Electronic Materials Ltd, UK) was then screen-printed onto the polyester substrate to cover the connections and define the active electrode areas, including that of the working electrode ( $3 \mathrm{~mm}$ diameter). After curing at $60^{\circ} \mathrm{C}$ for 30 minutes the SPEs are ready to be used. For experimental continuity, the on-board $\mathrm{Ag} / \mathrm{AgCl}$ reference and carbon counter electrodes for SPEs have been removed and replaced with an external SCE reference and Pt counter electrodes respectively (as mentioned previously). The SPEs have been electrochemically characterised previously and exhibit a heterogeneous electron transfer rate constant, $k_{\text {eff }}^{\mathrm{o}}$ of $c a .1 .08$ $\times 10^{-3} \mathrm{~cm} \mathrm{~s}^{-1}$ using $1 \mathrm{mM}$ hexaammineruthenium(III) chloride/ $0.1 \mathrm{M} \mathrm{KCl}^{30}$ The reproducibility and repeatability of the fabricated batches of electrodes were explored through comparison of cyclic voltammetric responses using $1 \mathrm{mM} \mathrm{Ru}\left(\mathrm{NH}_{3}\right)_{6}{ }^{2+/ 3+}$ in $0.1 \mathrm{M} \mathrm{KCl}$ supporting electrolyte. Analysis of the voltammetric data revealed the \% relative standard deviation (\% RSD) to correspond to no greater than $0.82 \%$ using the cathodic peak current.

The surfactant-exfoliated 2D-hBN utilised herein was fabricated in-house. Utilising a method reported previously by Kurapati et al.; ${ }^{31}$ bulk boron nitride was procured from Sigma Aldrich (used as received), after which it was sonicated in an aqueous solution (water, $\mathrm{pH}$ 7.6) containing sodium cholate, to induce liquid phase exfoliation. Interested readers are directed to the ESI $\uparrow$ for further details on the surfactant based liquid exfoliation, sonication and centrifugation methodology utilised. The resultant product comprised surfactant (sodium cholate) exfoliated 2D-hBN nanosheets sodium cholate $\left(2 \mathrm{~g} \mathrm{~L}^{-1}\right)$ in an aqueous solution. Note that where surfactant control experiments were utilised, a $2 \mathrm{~g} \mathrm{~L}^{-1}$ solution of sodium cholate was prepared and utilised in the absence of 2D-hBN (i.e. $2 \mathrm{D}$ material not present). The surfactant-free, 'pristine' 2D-hBN was commercially obtained from 'Graphene Supermarket' (Reading, MA, USA) and is known as 'Boron Nitride Pristine Flakes' comprised entirely of pristine 2D-hBN nanosheets dispersed in ethanol $\left(5.4 \mathrm{mg} \mathrm{L}^{-1}\right)$ that have not been oxidised, reduced or chemically modified in anyway. ${ }^{32}$ The pristine $2 \mathrm{D}$ hBN nanosheets are reported to have an average particle size (lateral) of $c a$. 50-200 nm, a thickness of between 1-5 monolayers in solution and a purity in the dry phase of $>99 \%$. $^{32}$

For experimental procedures/tests investigating the electrochemical detection of oxygen and capacitance studies, sulfuric acid solutions utilised were of the highest grade available from Sigma-Aldrich (99.999\%, double distilled for trace metal analysis) and was used at a concentration of $0.1 \mathrm{M}$. With regards to oxygen detection, the solution was subject to rigorous bubbling of $100 \%$ medicinal grade oxygen through $100 \mathrm{~mL}$ of the solution for 45 minutes, assuming this to be a completely saturated solution at room temperature as described by Gara. ${ }^{8}$ The concentration of oxygen was assumed to be $0.9 \mathrm{mM}$ according to previous reports using the same method. ${ }^{\mathbf{8 , 1 0}}$

Transmission electron microscopy (TEM) images were obtained using a $200 \mathrm{kV}$ primary beam under conventional brightfield conditions. The surfactant-exfoliated 2D-hBN sample was dispersed onto a holey-carbon film supported on a 300 mesh $\mathrm{Cu}$ TEM grid. Scanning electron microscopy (SEM) images and surface element analysis were obtained using a JEOL JSM5600LV model equipped with an energy-dispersive X-ray (EDX) microanalysis package. For Raman spectroscopy and X-ray diffraction (XRD) analysis, the surfactant-exfoliated 2D-hBN solution was drop-cast onto a supporting silicon wafer and glass slide respectively, then dried in air. Raman spectroscopy was performed using a 'Renishaw InVia' spectrometer with a confocal microscope $(\times 50$ objective $)$ spectrometer with an argon laser ( $514.3 \mathrm{~nm}$ excitation) at a very low laser power level $(0.8 \mathrm{~mW})$ to avoid any heating effects. Spectra were recorded using a 10 second exposure time for 3 accumulations. The XRD was performed using a "X'pert powder PANalytical model" with a copper source of $K_{\alpha}$ radiation of $1.54 \AA$ and $K_{\beta}$ radiation of $1.39 \AA$, using a thin sheet of nickel with an absorption edge of $1.49 \AA$ to absorb $K_{\beta}$ radiation. An Agilent $8453 \mathrm{UV}$-visible Spectroscopy System (equipped with a tungsten lamp assembly, G1315A, 8453 for absorption between $250 \mathrm{~nm}$ and $1500 \mathrm{~nm}$ and a deuterium lamp, 2140-0605 for absorption between $200 \mathrm{~nm}$ and $400 \mathrm{~nm}$ ) was used to obtain the absorption spectroscopy. The absorption spectra was analysed using the UV-Visible ChemStation software.

X-ray photoelectron spectroscopy (XPS) was used to analyse the $2 \mathrm{D}$ materials. XPS data were acquired using a bespoke ultrahigh vacuum system fitted with a Specs GmbH Focus 500 monochromated Al K $\alpha$ X-ray source, Specs GmbH PHOIBOS 150 $\mathrm{mm}$ mean radius hemispherical analyser with 9-channeltron detection, and a Specs GmbH FG20 charge neutralising electron gun. Survey spectra were acquired over the binding energy range 1100-0 eV using a pass energy of $50 \mathrm{eV}$ and high resolution scans were made over the $\mathrm{C} 1 \mathrm{~s}$ and $\mathrm{O}$ 1s lines using a pass energy of $20 \mathrm{eV}$. Under these conditions the full width at half maximum of the $\mathrm{Ag} 3 \mathrm{~d}_{5 / 2}$ reference line is $c a$. $0.7 \mathrm{eV}$. In each case, the analysis was an area-average over a region approximately $1.4 \mathrm{~mm}$ in diameter on the sample surface, using the 7 $\mathrm{mm}$ diameter aperture and lens magnification of $\times 5$. The energy scale of the instrument is calibrated according to ISO standard 15472, and the intensity scale is calibrated using an inhouse method traceable to the UK National Physical Laboratory. Data were quantified using Scofield photoelectric cross sections corrected for the energy dependencies of the electron attenuation lengths and the instrument transmission. Data interpretation was carried out using CasaXPS software.

\section{Results and discussion}

\section{Material characterisation}

$2 \mathrm{D}$ hexagonal boron nitride nanosheets $(2 \mathrm{D}-\mathrm{hBN})$ were fabricated as described in the Experimental section via surfactant exfoliation of bulk hexagonal boron nitride. Characterisation of the fabricated 2D-hBN was performed using TEM, SEM, XPS, 
XRD, UV-visible spectroscopy and Raman spectroscopy applied to provide a thorough physicochemical characterisation of the surfactant-exfoliated 2D-hBN. We first consider the structural characterisation of surfactant-exfoliated 2D-hBN with TEM. Typical TEM images are depicted in Fig. 1A and B where it can be observed that $2 \mathrm{D}-\mathrm{hBN}$ platelets have an average (lateral) particle size of $c a .200 \mathrm{~nm}$, which is in agreement to an independent literature report utilising the same fabrication methodology. ${ }^{31}$ It is also clear from the TEM images that the surfactant-exfoliated 2D-hBN exists in single layers. To investigate the layer thickness of surfactant-exfoliated 2D-hBN in solution, the UV-visible absorption spectrum methodology was employed (ESI Fig. 6†), where an optical band gap value of $5.61 \mathrm{eV}$ was found, indicating that $2-4$ layer $2 \mathrm{D}-\mathrm{hBN}$ is present in solution. ${ }^{33}$ This is also in good agreement with previous literature utilising the same fabrication methodology. ${ }^{31}$ Interested readers are referred to the $\mathrm{ESI} \dagger$ for full details of the optical band gap calculation and analysis. Fig. 1C shows a typical Raman spectra of the surfactant-exfoliated 2D-hBN, which gives rise to a characteristic Raman peak at 1366 $\mathrm{cm}^{-1} \cdot{ }^{34}$ The Raman peak is due to the $\mathrm{E}_{2 \mathrm{~g}}$ phonon mode and agrees well with prior literature reporting the fabrication of 2DhBN. ${ }^{35}$ Full width at half maximum (FWHM) peak analysis of the Raman spectra (Fig. 1C) exhibits a value that corresponds to $12.1 \mathrm{~cm}^{-1}$, which upon comparison to a recent report by Tran et $a l .{ }^{34}$ indicates that few to multilayer surfactant-exfoliated 2DhBN is present. Furthermore, SEM imaging (Fig. 1D) was obtained to assess how the 2D-hBN platelets prefer to reside upon the screen-printed electrode (SPEs) surfaces. XPS analysis was next performed on the surfactant-exfoliated 2D-hBN and is depicted in ESI Fig. 7 and $8 . \dagger$ Note that the analysis was conducted on a thin layer of $2 \mathrm{D}$-hBN, dried from a sodium cholate suspension on a Si (111) wafer fragment. The Si (111) wafer was sputter cleaned (prior to 2D-hBN deposition) using argon ions, in situ, within the vacuum of the XPS instrument to remove surface contaminants, thus allowing for the layer composition of surfactant-exfoliated $2 \mathrm{D}$-hBN to be calculated. The XPS spectra showed the presence of a single component at $191.1 \mathrm{eV}$ in the $\mathrm{B} 1 \mathrm{~s}$ spectrum and the $\mathrm{N}$ 1s spectrum exhibited a main peak at $398.7 \mathrm{eV}$ (ESI Fig. 8†), both in good agreement to previous literature. ${ }^{31}$ The stoichiometry of $1: 1$ for $\mathrm{B}: \mathrm{N}$ is noted and the binding energies for the $\mathrm{B} 1 \mathrm{~s}$ and $\mathrm{N} 1 \mathrm{~s}$ photoelectron

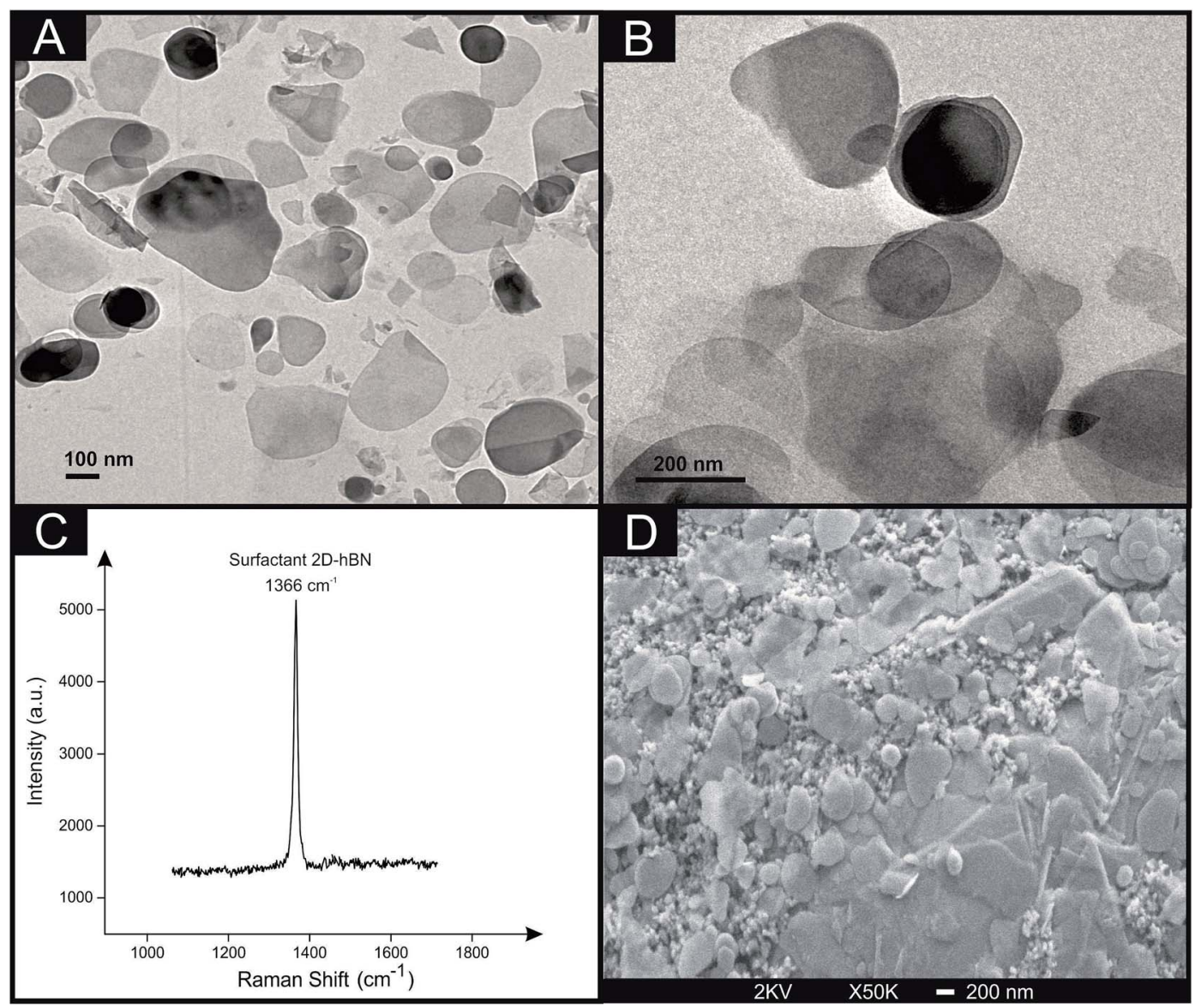

Fig. 1 Typical TEM images of surfactant-exfoliated 2D-hBN deposited onto a holey carbon film supported upon a Cu TEM grid (A), and at a higher magnification (B). Scale bars are $100 \mathrm{~nm}$ (A) and $200 \mathrm{~nm}$ (B). Images obtained using a 200 kV primary beam under bright-field conditions; (C), typical Raman spectra obtained for surfactant-exfoliated 2D-hBN (solid line) immobilised upon a supporting silicon wafer. (D) Typical SEM images for an SPE modified with $37.5 \mathrm{ng}$ surfactant-exfoliated 2D-hBN. 2D-hBN platelets are evident as small, disc-like shapes approximately $200 \mathrm{~nm}$ in size. 
peaks agree well with the expected values for $2 \mathrm{D}-\mathrm{hBN}$. ESI Table 1 exhibits the full surface composition of the dried surfactant-exfoliated 2D-hBN layer on Si (111) from the XPS analysis. The $\mathrm{C}$ and $\mathrm{O}$ present are a result of residuals from the sodium cholate surfactant used to disperse the $2 \mathrm{D}-\mathrm{hBN}$. The presence of a $\mathrm{C}-\mathrm{O}$ component in the $\mathrm{C} 1 \mathrm{~s}$ spectrum confirmed this, with the $\mathrm{Na}$ attributed to the sodium of the cholate structure (ESI Fig. 7†).

XRD was performed on the surfactant-exfoliated 2D-hBN with corresponding spectra depicted in ESI Fig. 9. $\dagger$ It is observed via XRD that surfactant-exfoliated 2D-hBN crystals are oriented in the (002) direction with a characteristic peak observed at $26.7^{\circ}$, in agreement with an independent literature report. ${ }^{36}$ The commercially procured surfactant-free (pristine) $2 \mathrm{D}$-hBN utilised in this study has also been fully characterised and interested readers are referred to the ESI for further information/details.

\section{The oxygen reduction reaction (ORR)}

Attention is now turned towards the oxygen reduction reaction (ORR), ${ }^{37}$ which is an important reaction that occurs at the cathode of PEM fuel cells. Due to its high kinetic parameter, research is directed towards reducing the potential at which the ORR occurs, ${ }^{38}$ with particular focus on 2D nanomaterials as a means to do this. ${ }^{39}$ Consequently, we explore $2 \mathrm{D}$-hBN towards the ORR using surfactant-exfoliated 2D-hBN immobilised upon SPEs (see ESI Fig. $5 \dagger$ ) where the underlying electrode serves to electrically wire/interconnect the $2 \mathrm{D}$-hBN materials.

First, unmodified, $37.5 \mathrm{ng}$ surfactant-exfoliated 2D-hBN and $10 \mu \mathrm{g}$ sodium cholate modified SPEs were explored towards the ORR within $0.1 \mathrm{M} \mathrm{H}_{2} \mathrm{SO}_{4}$. The $10 \mu \mathrm{g}$ sodium cholate modified SPE is a control experiment, which equates to the same volume of $37.5 \mathrm{ng}$ surfactant 2D-hBN drop-cast upon the electrode surface so that the sole effect of the sodium cholate on the ORR can be evaluated. It is important to note that the same sodium cholate was utilised in the exfoliation process of 2D-hBN (see Experimental section). One can observe, through inspection of Fig. 2, that the reduction potential of the ORR using an unmodified SPE occurs at -1.09 V. However upon

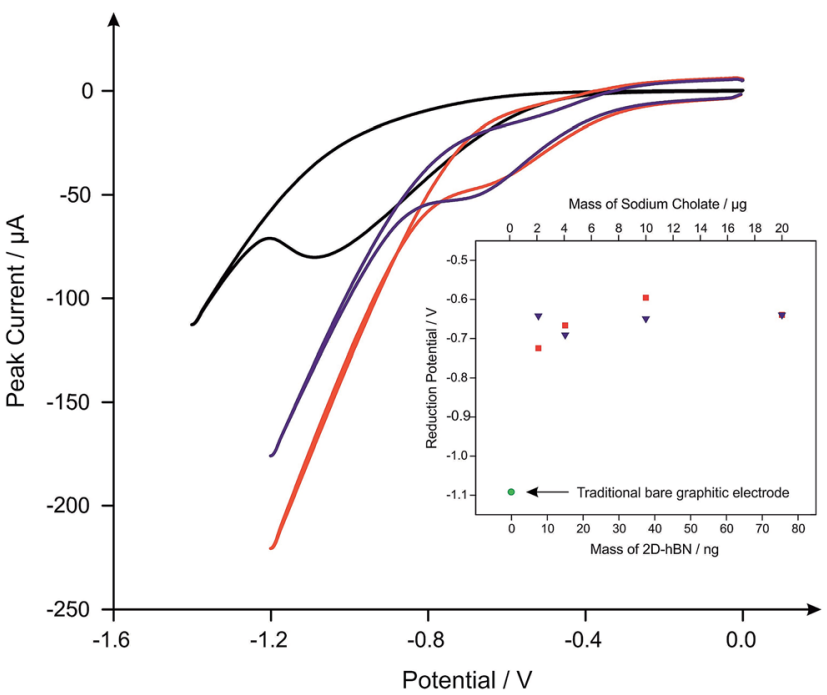

Fig. 2 Typical cyclic voltammograms recorded in an oxygen saturated $0.1 \mathrm{M} \mathrm{H}_{2} \mathrm{SO}_{4}$ solution using unmodified (black line), $37.5 \mathrm{ng}$ surfactantexfoliated 2D-hBN (red line) and $10 \mu$ g sodium cholate (on its own as a control, blue line) modified SPEs. Shown in the inset is analysis of the cyclic voltammograms in the form of a plot of oxygen reduction potential vs. mass of 2D-hBN (red squares) and sodium cholate (blue triangles) electrically wired upon SPEs. The green circle depicts an unmodified (bare) SPE. Scan rate: $100 \mathrm{mV} \mathrm{s}^{-1}$ (vs. SCE).

immobilisation of $37.5 \mathrm{ng}$ 2D-hBN, a decrease is exhibited in the potential required for the ORR to occur, shifting from -1.09 to $-0.59 \mathrm{~V}$ ( $v s$. SCE); hence a potential reduction of $0.50 \mathrm{~V}$ for the ORR to occur is observed (scan rate: $100 \mathrm{mV} \mathrm{s}^{-1}$ ), compared to the response of the bare underlying electrode. Next, control experiments were performed utilising sodium cholate modified SPEs towards the ORR to understand the role of surfactants in the electrochemical process. As a result, $10 \mu \mathrm{g}$ sodium cholate modified SPEs were utilised towards the ORR, and the voltammogram shown in Fig. 2 illustrates the potential required for the ORR to occur is $-0.65 \mathrm{~V}$ ( $v s$. SCE); thus a reduction in the potential for the ORR to occur of $0.44 \mathrm{~V}$ is observed (scan rate: $100 \mathrm{mV} \mathrm{s}^{-1}$ ) in comparison to an unmodified SPE. Hence, it is clear sodium cholate has a dominant role in the electrocatalytic

Table 1 A summary of the effect of increasing mass immobilisation of pristine 2D-hBN (75 ng), surfactant-exfoliated 2D-hBN (75 ng) and sodium cholate $(20 \mu \mathrm{g})$ upon SPEs towards; capacitance $\left(C_{\mathrm{WE}}\right) \mu \mathrm{F}$, specific capacitance $\left(C_{\mathrm{s}}\right) \mathrm{F} \mathrm{g}^{-1}$, areal capacitance $\left(C_{\mathrm{A}}\right) \mu \mathrm{F} \mathrm{cm}^{-2}$ and volumetric capacitance $\left(C_{V}\right) \mathrm{F} \mathrm{cm}^{-3}$. The current is held at $5.0 \mu \mathrm{A}$ throughout. Note capacitance measurements are obtained from charge/discharge profiles

\begin{tabular}{|c|c|c|c|c|c|c|}
\hline & \multicolumn{6}{|l|}{ Material } \\
\hline & $\begin{array}{l}\text { Unmodified } \\
\text { (bare) SPE }\end{array}$ & $\begin{array}{l}\text { Pristine } \\
\text { 2D-hBN }\end{array}$ & $\begin{array}{l}\text { Surfactant } \\
\text { 2D-hBN (2D-hBN) }\end{array}$ & $\begin{array}{l}\text { Surfactant 2D-hBN } \\
\text { (sodium cholate) }\end{array}$ & $\begin{array}{l}\text { Surfactant } 2 \mathrm{D} \text {-hBN } \\
(2 \mathrm{D}-\mathrm{hBN}+\text { sodium cholate })\end{array}$ & $\begin{array}{l}\text { Sodium } \\
\text { cholate }\end{array}$ \\
\hline Mass & $0.00030 \mathrm{~g}$ & $75 \mathrm{ng}$ & $75 \mathrm{ng}$ & $20 \mu \mathrm{g}$ & $2.0075 \times 10^{-5} \mathrm{~g}$ & $20 \mu \mathrm{g}$ \\
\hline $\begin{array}{l}\text { Capacitance } C_{\mathrm{WE}}(\mu \mathrm{F}) \\
\text { Specific capacitance } C_{\mathrm{s}}\left(\mathrm{F} \mathrm{g}^{-1}\right)\end{array}$ & 6.15 & 12.69 & 14.15 & 14.15 & 14.15 & 17.36 \\
\hline Method 3 & $\mathrm{~N} / \mathrm{A}$ & 87.12 & 106.73 & 0.40 & 0.40 & 1.12 \\
\hline Areal capacitance $C_{\mathrm{A}}\left(\mu \mathrm{F} \mathrm{cm}^{-2}\right)$ & 87.02 & 179.44 & 200.24 & 200.24 & 200.24 & 245.58 \\
\hline Volumetric capacitance $C_{\mathrm{V}}\left(\mathrm{F} \mathrm{cm}^{-3}\right)$ & 0.058 & 387.35 & 432.26 & 0.71 & 2.32 & 0.86 \\
\hline
\end{tabular}


effect shown towards the ORR upon the use of surfactantexfoliated 2D-hBN modified SPEs, given the reduction in the potential of $0.44 \mathrm{~V}$ when utilising a sodium cholate modified SPE. Corresponding voltammograms of nitrogen saturated $0.1 \mathrm{M} \mathrm{H}_{2} \mathrm{SO}_{4}$ utilising unmodified, $37.5 \mathrm{ng}$ surfactant 2D-hBN and $10 \mu \mathrm{g}$ sodium cholate modified SPEs are exhibited in ESI Fig. 16A, $\dagger$ where the absence of an oxygen signal is clearly apparent and the process observed in Fig. 2 is due to the electrochemical reduction of oxygen.

Following this, the deposition of various masses of surfactant-exfoliated 2D-hBN immobilised on SPEs and the effect upon the ORR is explored; again sodium cholate control investigations are also implemented (equating to the same volume of surfactant-exfoliated $2 \mathrm{D}-\mathrm{hBN}$ drop-cast onto the electrode surface). The effect of different masses and coverages is an overlooked parameter in the academic literature. As a result, SPEs were modified with surfactant-exfoliated 2D-hBN masses ranging from 7.5-75 ng; inset of Fig. 2 depicts a plot of the oxygen reduction peak potential $v s$. the mass of immobilised surfactant-exfoliated 2D-hBN. It is observed that increased amounts of surfactant-exfoliated 2D-hBN deposited upon SPEs leads to a decrease in the potential required for the ORR to occur in comparison to an unmodified SPE, with the activation potential shifting from $-1.09 \mathrm{~V}$ to $-0.73 \mathrm{~V},-0.67 \mathrm{~V},-0.59 \mathrm{~V}$ and $-0.64 \mathrm{~V}$ for $7.5,15,37.5$ and $75 \mathrm{ng}$ surfactant-exfoliated 2DhBN modified SPEs respectively. Thus a significant reduction in the activation potential for the ORR to occur is shown at various masses of immobilised 2D-hBN, with highest reduction $(0.50 \mathrm{~V})$ exhibited at $37.5 \mathrm{ng}$ modified 2D-hBN. Furthermore, sodium cholate control coverage studies were performed with the data presented in the inset of Fig. 2 which shows that the potential required for the ORR shifts from $-1.09 \mathrm{~V}$ to $-0.64 \mathrm{~V},-0.69 \mathrm{~V}$, $-0.65 \mathrm{~V}$ and $-0.64 \mathrm{~V}$ for, $2,4,10$ and $20 \mu \mathrm{g}$ sodium cholate modified SPEs respectively. This further demonstrates the dominant nature of the sodium cholate surfactant when utilised towards the ORR, which exhibits a similar electrocatalytic effect to that of the surfactant-exfoliated $2 \mathrm{D}$-hBN. Thus, it is suggested the significant electrocatalytic response obtained when utilising surfactant-exfoliated 2D-hBN towards the ORR, is inherently due to the surfactant, sodium cholate; such observations have not been reported before. The above data with 2D-hBN agrees with previous studies carried out by Brownson et al., ${ }^{40}$ whom observed that the electrocatalytic response of $2 \mathrm{D}$ materials such as graphene in various electroanalytical applications, should not assumed to be solely due to graphene itself, rather the effect of surfactants, such as sodium cholate, in the production of graphene dominate its electroanalytical response..$^{\mathbf{2 0 , 4 0}}$

We next consider the effect of surfactant-free (pristine) 2DhBN electrically wired upon SPEs towards the ORR. ESI Fig. $17 \dagger$ illustrates the voltammetric response obtained for pristine 2D-hBN modified SPEs (within 0.1 $\mathrm{M} \mathrm{H}_{2} \mathrm{SO}_{4}$ ), where it is clear that increasing immobilisation of pristine $2 \mathrm{D}-\mathrm{hBN}$ upon SPEs, of masses ranging from $54 \mathrm{ng}$ to $324 \mathrm{ng}$, reduces the potential required for the ORR to occur. Analysis of this is shown in ESI Fig. $17 \dagger$ inset, where it is revealed the potential required for the ORR to occur shifts from $-1.09 \mathrm{~V}$ (unmodified
SPE) to $-0.81 \mathrm{~V}\left(v s\right.$. SCE) at a scan rate of $100 \mathrm{mV} \mathrm{s}^{-1}$ upon increasing additions of pristine $2 \mathrm{D}-\mathrm{hBN}$, with the highest modification of $324 \mathrm{ng}$ pristine 2D-hBN, demonstrating a potential shift of $0.28 \mathrm{~V}$. Thus it is clear, pristine 2D-hBN exhibits electrocatalytic-type behaviour towards the ORR, however it is significantly inferior to that of surfactantexfoliated $2 \mathrm{D}-\mathrm{hBN}$, where a $0.5 \mathrm{~V}$ decrease in potential is observed. Therefore, it is inferred the surfactant, sodium cholate, utilised in the exfoliation of $2 \mathrm{D}-\mathrm{hBN}$ significantly enhances its electrochemical response. Corresponding voltammograms of nitrogen saturated $0.1 \mathrm{M} \mathrm{H}_{2} \mathrm{SO}_{4}$ utilising unmodified and 54-324 ng pristine 2D-hBN modified SPEs are exhibited in ESI Fig. 16B, $\uparrow$ where the absence of an oxygen signal is clearly apparent and the process observed in ESI Fig. $17 \dagger$ is due to the electrochemical reduction of oxygen.

A further consideration is the stability of the various $2 \mathrm{D}-\mathrm{hBN}$ modified layer(s) upon the electrode surface. We address this via the implementation of a stability experiment in which a 37.5 ng surfactant-exfoliated 2D-hBN, a $324 \mathrm{ng}$ pristine 2D-hBN and a $10 \mu \mathrm{g}$ sodium cholate modified SPE was subjected to 10 repeated cycles/scans in a $0.1 \mathrm{M} \mathrm{H}_{2} \mathrm{SO}_{4}$ solution at $100 \mathrm{mV} \mathrm{s}^{-1}$. This experiment simulates and recreates the appropriate timescale(s) of the voltammetric tests utilised within this work and shows that the cathodic $I_{\mathrm{p}}$ and potential remained stable, with only small alterations occurring in the recorded signal over 10 cycles (see ESI Fig. 18 and ESI Table $3 \dagger$ ), for the case of all modified electrodes. Clearly, at these representative coverages, the modified layer(s) are stable on the electrode surface and do not appear to fall off the electrode into the solution.

To gain further insights into the electrochemical mechanism, Tafel analysis was performed for unmodified, surfactantexfoliated 2D-hBN and sodium cholate modified SPEs, of masses ranging from $7.5-75 \mathrm{ng}$ and $2-20 \mu \mathrm{g}$ respectively. This analysis was also performed for pristine 2D-hBN modified SPEs of masses ranging from 108-324 ng. A plot of natural logarithm of the current $(\ln I) v s . E_{\mathrm{p}}$ was constructed for analysis of the voltammograms corresponding to the electrochemical reduction of oxygen using the following equation: $\partial \ln I / \partial E=\left(\alpha n^{\prime}\right) F /$ $R T$. The slope corresponds to $\partial \ln I / \partial E_{\mathrm{p}}$, where $\alpha$ is the electron transfer coefficient, $F$ is the Faraday constant and $n^{\prime}$ is the number of electrons transferred in the rate determining step, $R$ is the universal gas constant and $T$ is the absolute temperature. For an unmodified SPE, the $\alpha n^{\prime}$ value obtained was 0.25 . Within the Tafel region, the Nernstian potential was found to increase by $163.2 \mathrm{mV}$ for every order of magnitude of current. Conversely, for $7.5,15,37.5$ and $75 \mathrm{ng}$ surfactant-exfoliated 2D-hBN, $\alpha n^{\prime}$ values of $0.14,0.12,0.12$ and 0.12 were obtained, with a Nernstian potential increase of 93.24, 76.68, 81.13 and $82.12 \mathrm{mV}$ per order of magnitude of current respectively. Whereas, for 2, 4, 10 and $20 \mu \mathrm{g}$ sodium cholate modified SPEs $\alpha n^{\prime}$ values of 0.13 , $0.15,0.15$ and 0.14 were obtained, with a Nernstian potential increase of $88.37,96.70,98.39$ and $93.28 \mathrm{mV}$ per order of magnitude of current respectively. In the case of pristine $2 \mathrm{D}$ hBN modified SPEs of masses 108, 216 and $324 \mathrm{ng}, \alpha n^{\prime}$ values of $0.17,0.11$ and 0.11 were obtained, with a Nernstian potential increase of $113.47,75.63$ and $69.22 \mathrm{mV}$ per order of magnitude of current respectively. This infers that more current flows using 
surfactant-exfoliated 2D-hBN, sodium cholate and pristine $2 \mathrm{D}$ hBN modified electrodes in comparison to bare SPEs. Hence it is suggested the transfer of the first electron is electrochemically irreversible, so $n^{\prime}=1$ and $\alpha$ values of 0.25 and $0.14,0.12$, 0.12 and 0.12 are obtained for unmodified and 7.5, 15, 37.5 and $75 \mathrm{ng}$ surfactant-exfoliated 2D-hBN modified SPEs respectively. Furthermore, $\alpha$ values of $0.13,0.15,0.15$ and 0.14 were found to correspond to sodium cholate modified SPEs. Finally, the $\alpha$ values of 108, 216 and $324 \mathrm{ng}$ pristine 2D-hBN modified SPEs corresponded to $0.17,0.12$ and 0.11 respectively. The number of electrons transferred overall, $n$, was deduced using the following Randles-Ševćik equation for an irreversible electrochemical process: $I_{\mathrm{p}}=-0.496 n\left(\propto n^{\prime}\right)^{\frac{1}{2}} F A\left[\mathrm{O}_{2}\right](F v D / R T)^{\frac{1}{2}}$. A literature value of $0.9 \mathrm{mM}$ (ref. 10 and 41 ) is assumed for the oxygen saturated solution, and a literature diffusion coefficient value of $2.0 \times 10^{-5} \mathrm{~cm}^{2} \mathrm{~s}^{-1} . .^{42}$ A value of $n=2.66$ for an unmodified SPE was obtained whereas values of $n=3.45,3.04,2.91$ and 2.53 were deduced for 7.5, 15, 37.5 and $75 \mathrm{ng}$ surfactant-exfoliated 2D-hBN respectively and $n=2.79,2.75,2.91$ and 2.68 for 2,4 , 10 and $20 \mu \mathrm{g}$ sodium cholate modified SPEs. Pristine 2D-hBN modified SPEs of masses 108, 216 and $324 \mathrm{ng}$ resulted in values of $n=2.45,2.15$ and 1.90 respectively. This suggests the electrochemical reduction of oxygen using an unmodified, surfactant-exfoliated 2D-hBN, sodium cholate and pristine 2DhBN modified SPEs produces hydrogen peroxide $\left(\mathrm{H}_{2} \mathrm{O}_{2}\right)$ rather than the desirable product of $\mathrm{H}_{2} \mathrm{O}$. Previous studies have shown pristine 2D-hBN followed the two electron pathway mechanism and formed $\mathrm{H}_{2} \mathrm{O}_{2}$ when immobilised upon a gold substrate for the ORR. ${ }^{6}$ The observed average number of electrons transferred indicates that there is a similar mechanism for the ORR when utilising other $2 \mathrm{D}$ materials (graphene). ${ }^{\mathbf{1 0}}$

Next, hydrogen peroxide $\left(\mathrm{H}_{2} \mathrm{O}_{2}\right)$ yields were estimated for unmodified, surfactant-exfoliated 2D-hBN, sodium cholate and pristine 2D-hBN modified SPEs. First, the capacitance of an electrochemical process is estimated utilising: $C=I / v$, where $C$ is the capacitance, $I$ is the observed peak current at a certain potential and $v$ is the scan rate. Next, the charge is calculated using: $Q=C V$, where $Q$ is the charge and $V$ is the potential. Resultantly, $Q=n F N$ enables the amount of oxygen electrolysed in the reaction to be calculated, where $n$ is the number of electrons transferred, $F$ is the Faraday constant and $N$ is the number of moles of oxygen electrolysed. There is a $1: 1$ stoichiometric ratio of oxygen produced to $\mathrm{H}_{2} \mathrm{O}_{2}$, thus the concentration of oxygen electrolysed is theoretically the same as the concentration of $\mathrm{H}_{2} \mathrm{O}_{2}$ produced in the electrochemical reaction. Therefore the estimated $\mathrm{H}_{2} \mathrm{O}_{2}$ yields were calculated for an oxygenated $0.1 \mathrm{M} \mathrm{H}_{2} \mathrm{SO}_{4}$ solution at a fixed volume of 10 $\mathrm{mL}$ utilising unmodified, surfactant-exfoliated 2D-hBN, sodium cholate and pristine 2D-hBN modified SPEs of masses ranging from 7.5-75 $\mathrm{ng}, 2-20 \mu \mathrm{g}$ and $108-324 \mathrm{ng}$ respectively, at a scan rate of $100 \mathrm{mV} \mathrm{s}^{-1}$. It was estimated that the concentration of $\mathrm{H}_{2} \mathrm{O}_{2}$ electrolysed when utilising unmodified SPEs was $2.49 \mathrm{nM}$, whereas the surfactant-exfoliated 2D-hBN modified SPEs resulted in 1.41, 1.12, 0.98 and $0.99 \mathrm{nM}$ of $\mathrm{H}_{2} \mathrm{O}_{2}$ being produced for $7.5,15,37.5$ and $75 \mathrm{ng}$ of surfactant-exfoliated 2D-hBN respectively, thus a decrease. Comparatively, sodium cholate modified SPEs resulted in 1.08, 1.23, 1.20 and $1.09 \mathrm{nM}$ of $\mathrm{H}_{2} \mathrm{O}_{2}$ being produced for 2, 4, 10 and $20 \mu \mathrm{g}$ sodium cholate respectively. Finally, 2D-hBN modified SPEs resulted in 2.78, 3.01 and $3.22 \mathrm{nM}$ of $\mathrm{H}_{2} \mathrm{O}_{2}$ being produced for deposited masses of 108 , 216 and $324 \mathrm{ng}$ respectively. This suggests that surfactantexfoliated 2D-hBN, sodium cholate and pristine 2D-hBN modified SPEs follow a similar mechanism to unmodified SPEs for the ORR, given the minimal changes in peroxide yield.

A key factor in terms of the analytical performance of sensors is the inherent reproducibility of the response. The reproducibility of the electrode materials of interest was thus explored in acidic conditions with different masses of surfactant-exfoliated $2 \mathrm{D}$-hBN, sodium cholate and pristine $2 \mathrm{D}-\mathrm{hBN}$ deposited on the SPEs. A percentage relative standard deviation (\% RSD) in the analytical signal of $7.30 \%$ was observed for the unmodified SPEs $(N=3)$ at scan rate $100 \mathrm{mV} \mathrm{s}^{-1}$. This level of reproducibility is common for printed substrates and is a drawback for SPEs as reproducibility is slightly compromised for mass production. The 7.5, 15, 37.5, and $75 \mathrm{ng}$ surfactant-exfoliated 2D-hBN modified SPEs $\left(N=3 ; 100 \mathrm{mV} \mathrm{s}^{-1}\right)$ gave \% RSD values in the analytical signal of $0.35,0.47,1.21$, and $2.08 \%$, respectively. Furthermore, the 2, 4, 10, and $20 \mu \mathrm{g}$ pristine $2 \mathrm{D}$-hBN modified SPEs $\left(N=3 ; 100 \mathrm{mV} \mathrm{s}^{-1}\right)$ gave \% RSD values in the analytical signal of $0.44,0.71,1.09$, and $2.33 \%$, respectively. Finally, the $54,108,216$, and $324 \mathrm{ng}$ pristine 2D-hBN modified SPEs $(N=3$; $100 \mathrm{mV} \mathrm{s}^{-1}$ ) gave \% RSD values in the analytical signal of 1.81, $8.96,3.45$, and $12.59 \%$ respectively. The low \% RSD measurements obtained for the modified electrodes highlight the reproducibility of the drop-casting method for $2 \mathrm{D}-\mathrm{hBN}$ and sodium cholate upon SPEs.

If we consider the key observation of 2D-hBN utilised towards the ORR with surfactant fabrication compared to the surfactant-free approach, the former produces a greater reduction in the potential towards the ORR. There are two scenarios: the first is that different surface morphologies are evident. That is few layer $2 \mathrm{D}$-hBN via the surfactant protocol vs. few to multilayered pristine 2D-hBN, which leads towards its bulk form where the electrochemical properties of bulk h-BN are known to be inferior compared to $2 \mathrm{D}-\mathrm{hBN} .{ }^{43}$ Secondly, the surfactant is contributing to the response, as can be seen from control experiments, the response is due to the combination of the surfactant and 2D-hBN.

\section{Exploring 2D-hBN as a capacitive material}

Capacitance measurements of the $2 \mathrm{D}-\mathrm{hBN}$ material was next considered. In order to benchmark our approach, we first consider the cyclic voltammetric responses of our unmodified graphite based electrode material (SPE) utilising a two-electrode system in $0.1 \mathrm{M} \mathrm{H}_{2} \mathrm{SO}_{4}$ (scan rate $100 \mathrm{mV} \mathrm{s}^{-1}$ ) and observe the response exhibited upon immobilisation of pristine and surfactant-exfoliated 2D-hBN masses ranging from 7.5 to $75 \mathrm{ng}$. A sodium cholate control coverage study of masses ranging from 2$20 \mu \mathrm{g}$, equating to the same volume of surfactant 2D-hBN dropcast onto the electrode surface was also performed. ESI Fig. $19 \dagger$ depicts typical voltammograms obtained, where it is clear the capacitance of pristine 2D-hBN, surfactant-exfoliated 2D-hBN 
and sodium cholate modified SPEs is greatly superior to that of unmodified SPEs, indicated by the larger current passed. Analysis of these voltammograms is depicted in Fig. 3A, where a comparison study between the responses of unmodified, $75 \mathrm{ng}$ pristine 2D-hBN, $75 \mathrm{ng}$ surfactant-exfoliated 2D-hBN and $20 \mu \mathrm{g}$ sodium cholate modified SPEs is observed. It is clear that the unmodified SPEs display the lowest capacitance. Whereas immobilisation of $75 \mathrm{ng}$ pristine 2D-hBN upon an SPE increases the observed capacitance (due to an increase in current), comparatively, $75 \mathrm{ng}$ surfactant-exfoliated 2D-hBN immobilised upon an SPE exhibits a far superior capacitance in comparison to the pristine $2 \mathrm{D}-\mathrm{hBN}$ of the same mass. To rationalise this effect, a control study utilising $20 \mu \mathrm{g}$ sodium cholate immobilised upon an SPE was carried out, and it is established that sodium cholate modified SPEs per se offer the highest capacitance. This suggests the surfactant sodium cholate dominates the response.

To investigate this further, the capacitance was deduced for unmodified, pristine 2D-hBN, surfactant-exfoliated 2D-hBN and sodium cholate modified SPEs. The capacitance of the working electrode, $C_{\mathrm{WE}}$, was calculated from the corresponding voltammograms (see ESI Fig. 19†) using the following equation: $C_{\mathrm{WE}}=2\left(\frac{I}{v \Delta V}\right) \quad$ where $C_{\mathrm{WE}}$ is the capacitance exhibited by the working electrode, $I$ is the current, $v$ is the scan rate and $\Delta V$ is the difference in potential $\left(V_{2}-V_{1}\right)$ for each scan. Fig. 3B depicts the values obtained, where an unmodified SPE is found to have a capacitance $0.24 \mu \mathrm{F}$, whereas immobilisation of $75 \mathrm{ng}$ pristine $2 \mathrm{D}-\mathrm{hBN}, 75 \mathrm{ng}$ surfactant-exfoliated $2 \mathrm{D}-\mathrm{hBN}$ and $20 \mu \mathrm{g}$ sodium cholate upon an SPE increases the capacitance to 8.28, 13.55 and $21.07 \mu \mathrm{F}$ respectively. Hence, it is clear sodium cholate modified SPEs offer the highest capacitance in contrast to pristine and surfactant-exfoliated $2 \mathrm{D}-\mathrm{hBN}$ with a significant increase in capacitance observed in comparison to an unmodified SPE in each case. Furthermore, it is evident in Fig. $3 \mathrm{~B}$ that the capacitance of surfactant-exfoliated 2D-hBN decreases upon $75 \mathrm{ng}$ 2D-hBN immobilisation on an SPE, with the highest value of $14.9 \mu \mathrm{F}$ value observed upon $37.5 \mathrm{ng} 2 \mathrm{D}$-hBN modification. Contrastingly, the capacitance of sodium cholate modified SPEs continue to increase upon each modification. Therefore the response exhibited when utilising surfactant-exfoliated 2D-hBN modified SPEs, should not assumed to be solely due to 2D-hBN, rather the surfactant used in the production of $2 \mathrm{D}-\mathrm{hBN}$ is dominating and improving the response of $2 \mathrm{D}-\mathrm{hBN}$, an effect comparable to that which was observed previously in the detection of oxygen. A summary of the results are shown in ESI Table $4 . \dagger$

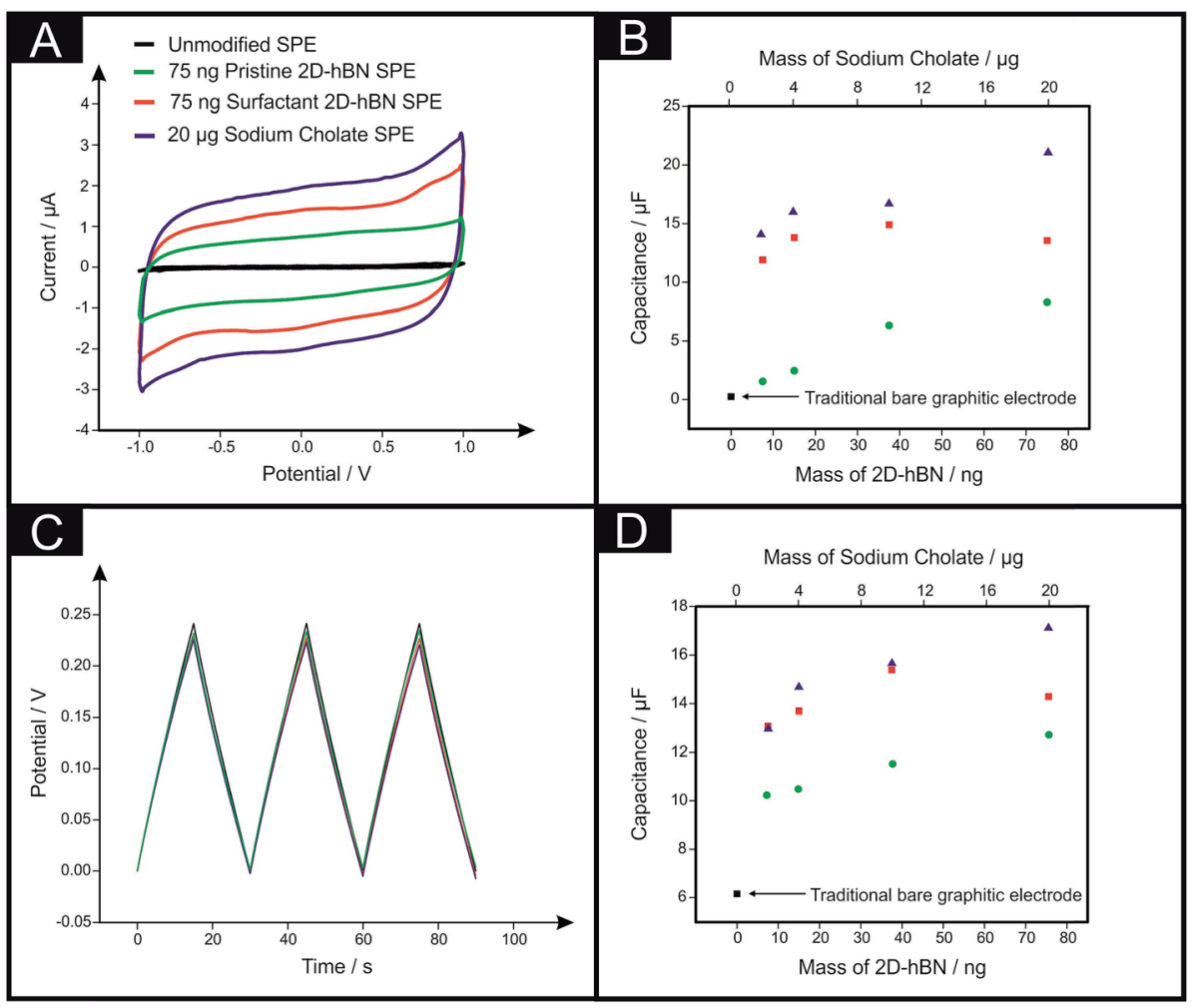

Fig. 3 Typical cyclic voltammograms (A) recorded in $0.1 \mathrm{M} \mathrm{H}_{2} \mathrm{SO}_{4}$ with unmodified (black line) and $75 \mathrm{ng}$ pristine 2D-hBN (green line), $75 \mathrm{ng}$ surfactant-exfoliated 2D-hBN (red line) and $20 \mu \mathrm{g}$ sodium cholate (blue line) modified SPEs. Scan rate: $100 \mathrm{mV} \mathrm{s}^{-1}$. (B) Analysis of voltammograms (ESI Fig. 19†) illustrates the effects of SPEs modified with masses of pristine 2D-hBN (green) and surfactant-exfoliated 2D-hBN (red), mass range: $7.5,15,37.5$ and $75 \mathrm{ng}$ along with sodium cholate (blue) mass range: $2,4,10$ and $20 \mu \mathrm{g}$ vs. the capacitance ( $\mu \mathrm{F}$ ). (C) Typical charge/ discharge profiles recorded in $0.1 \mathrm{M} \mathrm{H}_{2} \mathrm{SO}_{4}$ with unmodified (black) $75 \mathrm{ng}$ pristine 2D-hBN (green), 75 ng surfactant-exfoliated 2D-hBN (red) and $20 \mu \mathrm{g}$ sodium cholate (blue) modified SPEs. (D) Analysis of charge/discharge profiles illustrates the effects of SPEs modified with masses of pristine $2 \mathrm{D}-\mathrm{hBN}$ (green) and surfactant-exfoliated 2D-hBN (red), mass range: 7.5, 15, 37.5 and $75 \mathrm{ng}$ along with sodium cholate (blue) mass range: $2,4,10$ and $20 \mu \mathrm{g}$, vs. the capacitance $(\mu \mathrm{F})$. 
Next, a galvanostatic charge/discharge study was performed using a two-electrode approach to compliment the setup utilised for supercapacitors within the field. Fig. 3C illustrates the charge/discharge profiles obtained for unmodified, $75 \mathrm{ng}$ pristine 2D-hBN, $75 \mathrm{ng}$ surfactant-exfoliated $2 \mathrm{D}-\mathrm{hBN}$ and $20 \mu \mathrm{g}$ sodium cholate modified SPEs. Measurements were undertaken upon utilisation of the circuit with $300 \mu \mathrm{F}$ parallel capacitors (see the Experimental section), ${ }^{19}$ to improve the linearity of the slope of the charge/discharge profiles, thus allowing for a more accurate analysis of the capacitive characteristics to be established. ${ }^{\mathbf{1 9 , 4 4}}$ Interested readers are referred to the $\mathrm{ESI} \dagger$ for further information. It is shown in Fig. 3C, that the potential of the charge/discharge profiles incrementally decrease upon immobilisation of $75 \mathrm{ng}$ pristine 2D-hBN, $75 \mathrm{ng}$ surfactant-exfoliated $2 \mathrm{D}-\mathrm{hBN}$ and the highest reduction is observed upon a $20 \mu \mathrm{g}$ modified SPE, in comparison to an unmodified SPE, suggesting an increase in capacitance. To evaluate this further, the capacitance, $C_{\mathrm{WE}}$, was calculated from the corresponding galvanostatic charge/discharge profiles using the following equation: ${ }^{19} \quad C_{\mathrm{WE}}=\frac{1}{2}\left[\frac{I}{\mathrm{~d} V / \mathrm{d} t}-C_{\text {known }}\right]$ where $C_{\mathrm{WE}}$ is the capacitance exhibited by the working electrode, $I$ is the current, $(\mathrm{d} V / \mathrm{d} t)$ is the slope of potential versus time and $C_{\text {known }}$ is the capacitance of the parallel circuit. $^{\mathbf{1 9 , 4 4}}$ Analysis of charge/ discharge profiles of unmodified SPEs, pristine and surfactant-exfoliated 2D-hBN modified SPEs, (masses ranging from 7.5 to $75 \mathrm{ng}$ ) and sodium cholate modified SPEs of masses ranging from 2-20 $\mu \mathrm{g}$ are exhibited in Fig. 3D. An unmodified SPE is found to have a capacitance of $6.15 \mu \mathrm{F}$, whereas immobilisation of $75 \mathrm{ng}$ pristine 2D-hBN, $75 \mathrm{ng}$ surfactant-exfoliated 2D-hBN and $20 \mu \mathrm{g}$ sodium cholate upon an SPE increases the capacitance to $12.68,14.20$ and $17.36 \mu \mathrm{F}$ respectively, at a current of $5.0 \mu \mathrm{A}$, (which provided the best signal to noise ratio). Hence, the charge/discharge profiles offer a similar trend to that of the voltammetry method when measuring the capacitance and it is again clear sodium cholate modified SPEs offer the highest capacitance in contrast to pristine and surfactant-exfoliated 2DhBN with a significant increase in capacitance observed when compared to an unmodified SPE in each modified case. Furthermore, it is again evident in Fig. 3D that the capacitance of surfactant-exfoliated 2D-hBN decreases upon $75 \mathrm{ng} 2 \mathrm{D}-\mathrm{hBN}$ immobilisation on an SPE, with the highest value of $15.39 \mu \mathrm{F}$ value observed upon $37.5 \mathrm{ng} 2 \mathrm{D}$-hBN modification. Whereas, sodium cholate modified SPEs continue to exhibit an increase in capacitance upon each modification. Pristine 2D-hBN modification offers the least favourable increase in capacitance. Thus, it is established, from charge/discharge profiles and voltammograms, the surfactant, sodium cholate, used in the exfoliation of 2D-hBN is dominating the observed beneficial response towards capacitance measurements and this effect is not due solely to 2D-hBN (a summary of the results are reported in Table 1). Additionally, a capacitive retention study over 500 charge/discharge cycles is depicted within ESI Fig. 20, $\dagger$ demonstrating the incorporation of sodium cholate in the fabrication process of $2 \mathrm{D}$-hBN results in a negative effect upon its' cycling stability, with the highest retention shown utilising pristine $2 \mathrm{D}-\mathrm{hBN}$.
Attention was next turned towards obtaining the specific capacitance $\left(C_{\mathrm{s}}\right)$ values of unmodified, pristine $2 \mathrm{D}-\mathrm{hBN}$, surfactant-exfoliated $2 \mathrm{D}$-hBN and sodium cholate modified SPEs from said charge/discharge profiles. Current literature utilises an array of methods in the calculation of $C_{\mathrm{s}}$, however the differences observed for each method are not reported. Thus, in this work a diverse range of methods were utilised to calculate the $C_{\mathrm{s}}$ values and Table 1 exhibits the differences observed. Method 1 is the typical analysis of electrode performance, which assumes that all of the performance is a result of the added material; method 2 evaluates the improved specific capacitance as a result of the material added to the electrode by reducing by the capacitive value of the unmodified electrode; and method 3 indicates the specific capacitance associated with the drop-cast material only. From these equations, $C_{\mathrm{WE}(\text { modified) }}$ is the capacitance $(F)$ of the material immobilised upon an SPE, $C_{\mathrm{WE} \text { (unmodified) }}$ is the capacitance $(F)$ of the unmodified (bare) SPE. Furthermore, $m_{1}$ is the mass $(\mathrm{g})$ of material immobilised upon an SPE and $m_{2}$ is the mass (g) of an unmodified (bare) SPE.

Method 1:

$$
C_{\mathrm{s}}=\frac{C_{\mathrm{WE}(\text { modified })}}{m_{1}}
$$

Method 2:

$$
C_{\mathrm{s}}=\left(\frac{C_{\mathrm{WE}(\text { modified })}}{m_{1}}\right)-\left(\frac{C_{\mathrm{WE}(\text { unmodified })}}{m_{2}}\right)
$$

Method 3:

$$
C_{\mathrm{s}}=\frac{C_{\mathrm{WE} \text { (modified) }}-C_{\mathrm{WE}(\text { unmodified })}}{m_{1}}
$$

The hope is that standardisation of measuring the capacitance of materials can be achieved in the academic literature. Table 1 exhibits the $C_{\mathrm{s}}$ values obtained for unmodified, $75 \mathrm{ng}$ pristine $2 \mathrm{D}-\mathrm{hBN}, 75 \mathrm{ng}$ surfactant-exfoliated $2 \mathrm{D}-\mathrm{hBN}$ and $20 \mu \mathrm{g}$ sodium cholate modified SPEs. The $C_{\mathrm{s}}$ values for $75 \mathrm{ng}$ pristine 2D-hBN immobilised upon an SPE obtained are 169.15, 169.13 and $87.12 \mathrm{~F} \mathrm{~g}^{-1}$ when utilising methods 1,2 and 3 respectively. Whereas, surfactant-exfolaited 2D-hBN, for which $75 \mathrm{ng}$ 2D-hBN is specifically analysed offers $C_{\mathrm{s}}$ values of $188.76,188.74$ and 106.73 $\mathrm{F} \mathrm{g}^{-1}$ for methods 1,2 and 3 respectively. Thus, an increase $C_{\mathrm{s}}$ in comparison to pristine $2 \mathrm{D}-\mathrm{hBN}$ is observed. Comparatively, when evaluating surfactant-exfoliated 2D-hBN for which $20 \mu \mathrm{g}$ sodium cholate is specifically analysed, $C_{\mathrm{s}}$ values of $0.71,0.69$ and 0.40 are observed when utilising method 1, 2 and 3 respectively. Thus it is apparent when investigating surfactant-exfoliated $2 \mathrm{D}$-hBN, there is a significant difference in $C_{\mathrm{s}}$ value when analysing either $2 \mathrm{D}$-hBN or sodium cholate. Note each case has the same capacitance value (14.15 $\mu \mathrm{F})$. The combination of $75 \mathrm{ng} 2 \mathrm{D}-\mathrm{hBN}$ and $20 \mu \mathrm{g}$ sodium cholate towards obtaining a $C_{\mathrm{s}}$ value for the surfactantexfoliated $2 \mathrm{D}$-hBN was also investigated. As a result, $C_{\mathrm{s}}$ values of $0.71,0.68$ and $0.40 \mathrm{~F} \mathrm{~g}^{-1}$ are observed when utilising methods 1,2 and 3 respectively. Thus, due to the lower mass of the $2 \mathrm{D}$ - 
$\mathrm{hBN}$, a higher $C_{\mathrm{s}}$ value is obtained in comparison to the sodium cholate, indicating the importance of evaluating all materials utilised in the exfoliation of 2D-hBN. Finally, a control study utilising $20 \mu \mathrm{g}$ sodium cholate per se was investigated. Table 1 exhibits the $C_{\mathrm{s}}$ values obtained of $0.87,1.72$ and $1.12 \mathrm{~F} \mathrm{~g} \mathrm{~g}^{-1}$ for methods, 1, 2 and 3 respectively. These values incrementally increase in comparison to $20 \mu \mathrm{g}$ sodium cholate in $2 \mathrm{D}-\mathrm{hBN}$, due to the higher capacitance $(17.36 \mu \mathrm{F})$. However, as explained above, due to the higher mass of sodium cholate in comparison to $2 \mathrm{D}-\mathrm{hBN}$, a lower $C s$ value is obtained. Therefore, it is important to consider both materials (2D-hBN and sodium cholate) when investigating the $C_{\mathrm{s}}$ values of surfactantexfoliated $2 \mathrm{D}-\mathrm{hBN}$ rather than solely $2 \mathrm{D}-\mathrm{hBN}$, as it is clear that significant differences are observed due to the mass implemented when calculating the $C_{\mathrm{s}}$. Indeed, the utilisation of a $7.5 \mathrm{ng}$ surfactant-exfoliated 2D-hBN modified SPE (lowest mass) results in the highest $C_{\mathrm{s}}$ value $\left(1745 \mathrm{~F} \mathrm{~g}^{-1}\right)$ see ESI Table 5 and ESI Fig. 21. $\dagger$ Consequently, the comparison of the capacitance $\left(C_{\mathrm{WE}}\right)$ values alone demonstrate more realistic properties when considering industrial applications. Furthermore, the volumetric and areal capacitance were also calculated for the 75 ng pristine $75 \mathrm{ng} 2 \mathrm{D}$-hBN, surfactant-exfoliated 2D-hBN and 20 $\mu \mathrm{g}$ sodium cholate, the values of which can be found in Table 1. A full summary for all masses of pristine 2D-hBN, surfactantexfoliated 2D-hBN and sodium cholate towards measurements of capacitance $\left(\mathrm{C}_{\mathrm{WE}}\right)$, specific capacitance $\left(C_{\mathrm{s}}\right)$, volumetric $\left(C_{\mathrm{V}}\right)$ and areal capacitance $\left(C_{\mathrm{A}}\right)$ are summarized within ESI Table $5 . \dagger$

\section{Conclusions}

We have demonstrated for the first time that surfactantexfoliated 2D-hBN exhibits an electrocatalytic effect towards the ORR when immobilised upon graphitic substrate (SPE). A potential reduction of $c a$. $0.50 \mathrm{~V}$ was observed upon immobilisation of $37.5 \mathrm{ng}$ surfactant-exfoliated 2D-hBN on an SPE, in comparison to its bare equivalent. However, through the utilisation of control coverage studies, the large electrocatalytic effect is highly dependent upon the surfactant (sodium cholate) utilised, with a potential reduction of $c a$. $0.44 \mathrm{~V}$ observed when utilising just sodium cholate (no 2D-hBN) modified SPEs.

Furthermore, it was demonstrated for the first time surfactant-exfoliated $2 \mathrm{D}$-hBN is an effective electrochemical supercapacitor material, with a specific capacitance value of up to $1745 \mathrm{~F} \mathrm{~g}^{-1}$ obtained for $7.5 \mathrm{ng}$ surfactant-exfoliated 2D-hBN modified SPEs (ESI Fig. 21 and ESI Table $5 \dagger$ ). Furthermore, a diverse range of methods are introduced and utilised to calculate the specific capacitance, a substantially overlooked and misinterpreted parameter within the literature allowing standardisation in the academic literature to be achieved.

In both electrochemical systems, the surfactant was the overall dominating factor in the observed 2D-hBN electrocatalytic and capacitive $(\mu \mathrm{F})$ response. Thus, future research involving surfactant-exfoliated 2D-hBN should consider the electrochemical behaviour of the surfactant alone to allow the true de-convolution of the electrochemical response of $2 \mathrm{D}-\mathrm{hBN}$; without this control experiment, $2 \mathrm{D}-\mathrm{hBN}$ can be wrongly attributed to be electrochemically catalytic/active when actually it is the surfactant itself being the more active material.

\section{Acknowledgements}

The authors acknowledge funding from the Engineering and Physical Sciences Research Council (Reference: EP/N001877/1) and a British Council Institutional Grant Link (No. 172726574).

\section{References}

1 D. A. C. Brownson, D. K. Kampouris and C. E. Banks, J. Power Sources, 2011, 196, 4873-4885.

2 D.-W. Wang and D. Su, Energy Environ. Sci., 2014, 7, 576-591.

3 D. Higgins, P. Zamani, A. Yu and Z. Chen, Energy Environ. Sci., 2016, 9, 357-390.

4 H. Jin, H. Zhang, H. Zhong and J. Zhang, Energy Environ. Sci., 2011, 4, 3389-3394.

5 A. Morozan, B. Jousselme and S. Palacin, Energy Environ. Sci., 2011, 4, 1238-1254.

6 K. Uosaki, G. Elumalai, H. Noguchi, T. Masuda, A. Lyalin, A. Nakayama and T. Taketsugu, J. Am. Chem. Soc., 2014, 136, 6542-6545.

7 O. T. Holton and J. W. Stevenson, Platinum Met. Rev., 2013, 57, 259-271.

8 M. Gara and R. G. Compton, New J. Chem., 2011, 35, 26472652.

9 C. Song and J. Zhang, in PEM Fuel Cell Electrocatalysts and Catalyst Layers, ed. J. Zhang, Springer, London, 2008, ch. 2, pp. 89-134, DOI: 10.1007/978-1-84800-936-3_2.

10 E. P. Randviir and C. E. Banks, Electroanalysis, 2014, 26, 7683.

11 D. Geng, Y. Chen, Y. Chen, Y. Li, R. Li, X. Sun, S. Ye and S. Knights, Energy Environ. Sci., 2011, 4, 760-764.

12 U. Kramm, in Encyclopedia of Applied Electrochemistry, ed. G. Kreysa, K.-i. Ota and R. Savinell, Springer, New York, 2014, ch. 204, pp. 909-918, DOI: 10.1007/978-1-4419-6996-5_204.

13 D. Qazzazie, M. Beckert, R. Mülhaupt, O. Yurchenko and G. Urban, J. Phys.: Conf. Ser., 2014, 557, 012009.

14 S. J. Rowley-Neale, J. M. Fearn, D. A. C. Brownson, G. C. Smith, X. Ji and C. E. Banks, Nanoscale, 2016, 8, 14767-14777.

15 K. Zhao, W. Gu, L. Zhao, C. Zhang, W. Peng and Y. Xian, Electrochim. Acta, 2015, 169, 142-149.

16 J. Zhao and Z. Chen, J. Phys. Chem. C, 2015, 119, 2634826354.

17 A. F. Khan, E. P. Randviir, D. A. C. Brownson, X. Ji, G. C. Smith and C. E. Banks, Electroanalysis, 2016, DOI: 10.1002/elan.201600462.

18 C.-C. Hu, K.-H. Chang, M.-C. Lin and Y.-T. Wu, Nano Lett., 2006, 6, 2690-2695.

19 D. K. Kampouris, X. Ji, E. P. Randviir and C. E. Banks, RSC Adv., 2015, 5, 12782-12791.

20 D. A. C. Brownson and C. E. Banks, Chem. Commun., 2012, 48, 1425-1427.

21 E. Iyyamperumal, S. Wang and L. Dai, ACS Nano, 2012, 6, 5259-5265. 
22 Z.-S. Wu, A. Winter, L. Chen, Y. Sun, A. Turchanin, X. Feng and K. Müllen, Adv. Mater., 2012, 24, 5130-5135.

23 K. Wang, L. Li, T. Zhang and Z. Liu, Energy, 2014, 70, 612617.

24 V. Sahu, S. Grover, B. Tulachan, M. Sharma, G. Srivastava, M. Roy, M. Saxena, N. Sethy, K. Bhargava, D. Philip, H. Kim, G. Singh, S. K. Singh, M. Das and R. K. Sharma, Electrochim. Acta, 2015, 160, 244-253.

25 L. Niu, Z. Li, W. Hong, J. Sun, Z. Wang, L. Ma, J. Wang and S. Yang, Electrochim. Acta, 2013, 108, 666-673.

26 T. Gao, L.-j. Gong, Z. Wang, Z.-k. Yang, W. Pan, L. He, J. Zhang, E.-c. Ou, Y. Xiong and W. Xu, Mater. Lett., 2015, 159, 54-57.

27 E. Blanco, C. W. Foster, L. R. Cumba, D. R. do Carmo and C. E. Banks, Analyst, 2016, 141, 2783.

28 L. R. Cumba, C. W. Foster, D. A. C. Brownson, J. P. Smith, J. Iniesta, B. Thakur, D. R. do Carmo and C. E. Banks, Analyst, 2016, 141, 2791.

29 E. P. Randviir, D. A. C. Brownson, J. P. Metters, R. O. Kadara and C. E. Banks, Phys. Chem. Chem. Phys., 2014, 16, 45984611.

30 J. P. Metters, R. O. Kadara and C. E. Banks, Sens. Actuators, B, 2012, 169, 136-143.

31 R. Kurapati, C. Backes, C. Ménard-Moyon, J. N. Coleman and A. Bianco, Angew. Chem., Int. Ed., 2016, 55, 5506-5511.

32 Graphene Supermarket, https://graphene-supermarket.com, accessed, 04/01/2016.
33 L. Song, L. Ci, H. Lu, P. B. Sorokin, C. Jin, J. Ni, A. G. Kvashnin, D. G. Kvashnin, J. Lou, B. I. Yakobson and P. M. Ajayan, Nano Lett., 2010, 10, 3209-3215.

34 T. T. Tran, K. Bray, M. J. Ford, M. Toth and I. Aharonovich, Nat. Nanotechnol., 2016, 11, 37-41.

35 R. V. Gorbachev, I. Riaz, R. R. Nair, R. Jalil, L. Britnell, B. D. Belle, E. W. Hill, K. S. Novoselov, K. Watanabe, T. Taniguchi, A. K. Geim and P. Blake, Small, 2011, 7, 465468.

36 G. R. Bhimanapati, D. Kozuch and J. A. Robinson, Nanoscale, 2014, 6, 11671-11675.

37 S. Gadipelli, T. Zhao, S. A. Shevlin and Z. Guo, Energy Environ. Sci., 2016, 9, 1661-1667.

38 I. E. L. Stephens, A. S. Bondarenko, U. Gronbjerg, J. Rossmeisl and I. Chorkendorff, Energy Environ. Sci., 2012, 5, 6744-6762.

39 L. Lai, J. R. Potts, D. Zhan, L. Wang, C. K. Poh, C. Tang, H. Gong, Z. Shen, J. Lin and R. S. Ruoff, Energy Environ. Sci., 2012, 5, 7936-7942.

40 D. A. C. Brownson, J. P. Metters, D. K. Kampouris and C. E. Banks, Electroanalysis, 2011, 23, 894-899.

41 T. Kaskiala, Miner. Eng., 2002, 15, 853-857.

42 P. Han and D. M. Bartels, J. Phys. Chem., 1996, 100, 55975602.

43 Y. Xue, Q. Liu, G. He, K. Xu, L. Jiang, X. $\mathrm{Hu}$ and J. $\mathrm{Hu}$, Nanoscale Res. Lett., 2013, 8, 1-7.

44 M. P. Down, C. W. Foster, X. Ji and C. E. Banks, RSC Adv., 2016, 6, 81130-81141. 